\title{
Estimation of turbulence dissipation rate and its variability from sonic anemometer and wind Doppler lidar during the XPIA field campaign
}

Nicola Bodini et al.

Correspondence to: Nicola Bodini (nicola.bodini@colorado.edu)

The copyright of individual parts of the supplement might differ from the CC BY 4.0 License. 
1. Second-order structure function from sonic anemometer measurements

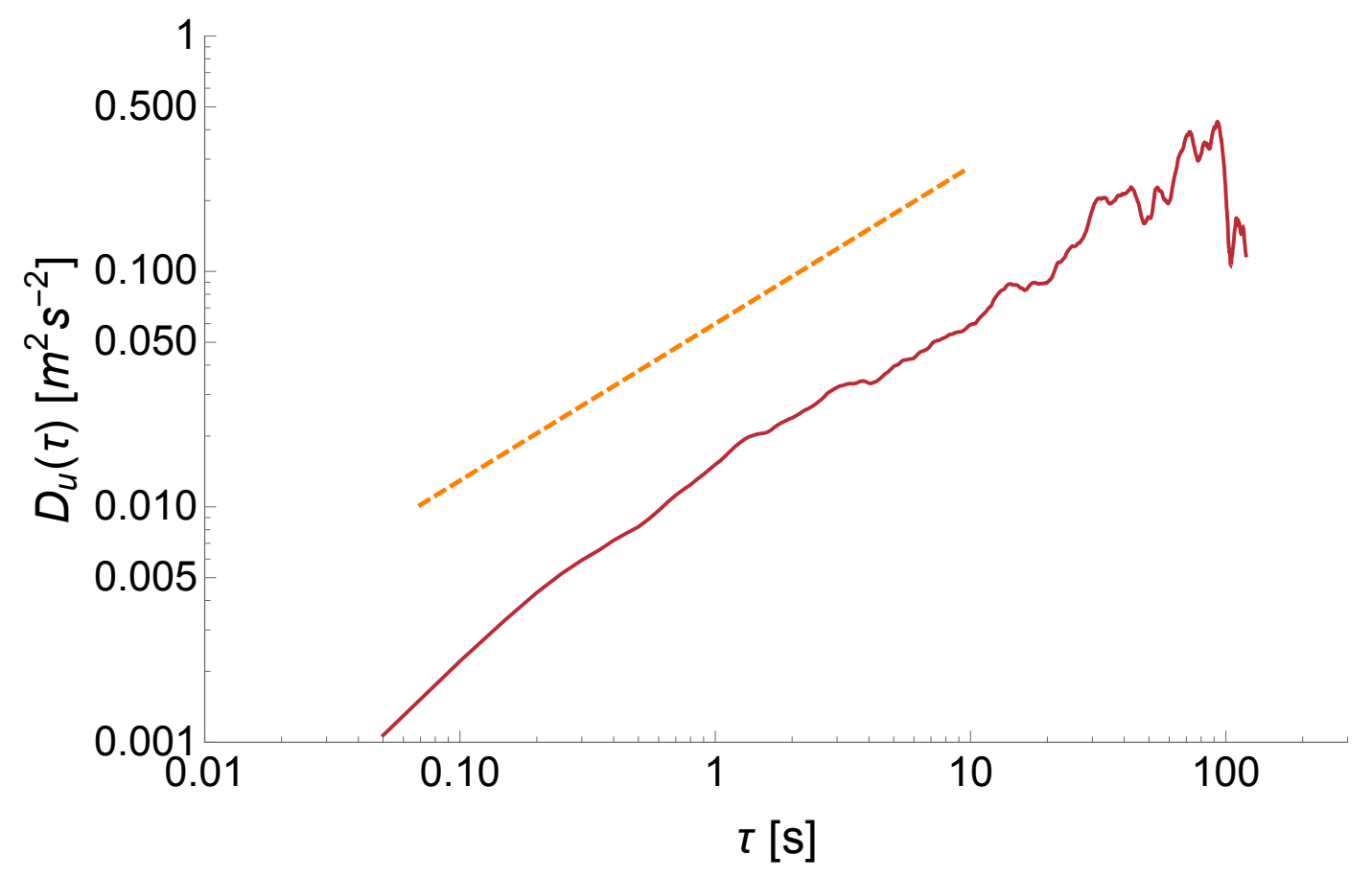

Figure S1: Second-order structure function from sonic anemometer measurements at 100m AGL, for March 2015, 19:58 UTC. The dashed line represents the theoretical Kolmogorov's inertial range slope $\tau^{2 / 3}$. 


\section{Comparison of turbulence dissipation rate from sonic anemometers in opposite tower booms}

(a)

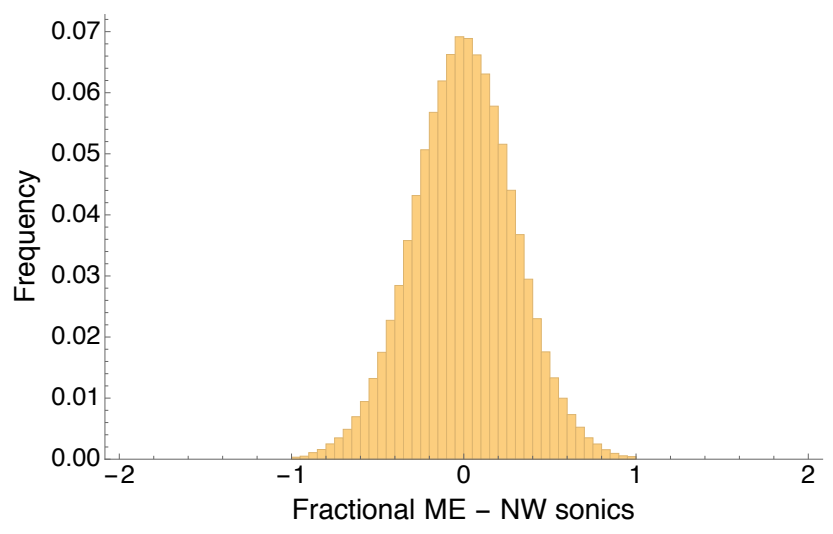

(b)

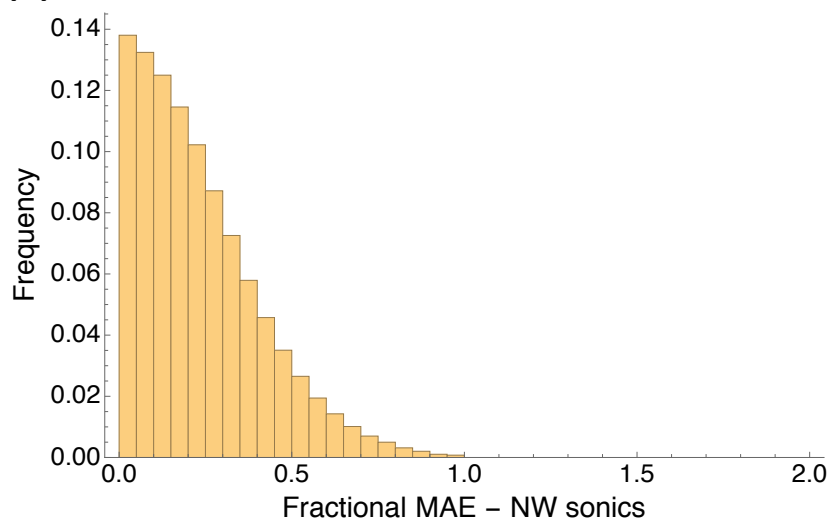

Figure S2: (a) histogram of the fractional median error between turbulence dissipation rate calculated from the sonic anemometers on the northwest booms and the average dissipation from both the boom directions. Results for the sonic anemometers on the southeast booms are similar. (b) as in (a), but median absolute error. Raw values of $\epsilon$ are used. 


\section{Variation of the sample size with height for the Halo Streamline lidar}

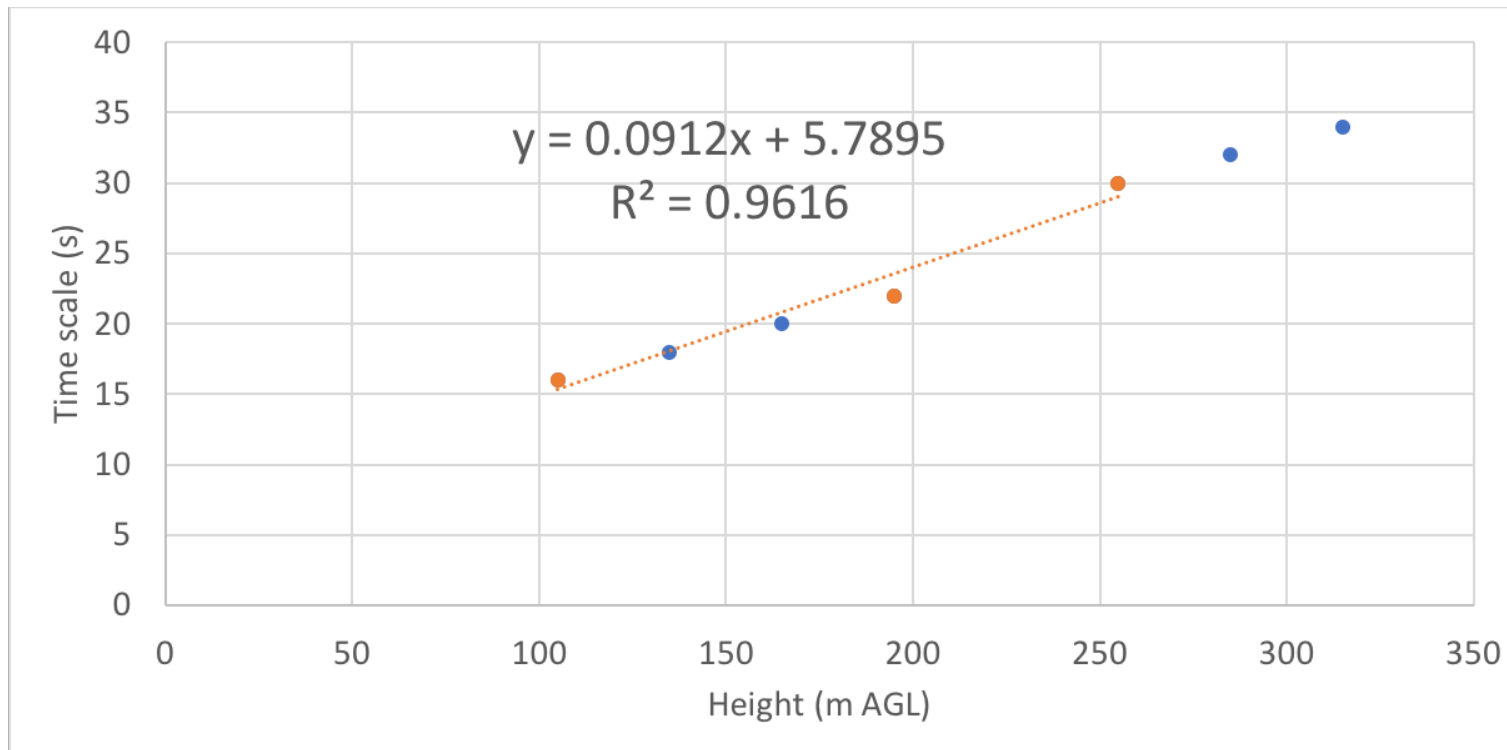

Figure S3: variability of the appropriate sample size with height for the Halo Streamline lidar, during stable conditions. The best-fit is performed by using the orange dots only, as these correspond to heights with a match with the levels of the sonic anemometers on the BAO tower.

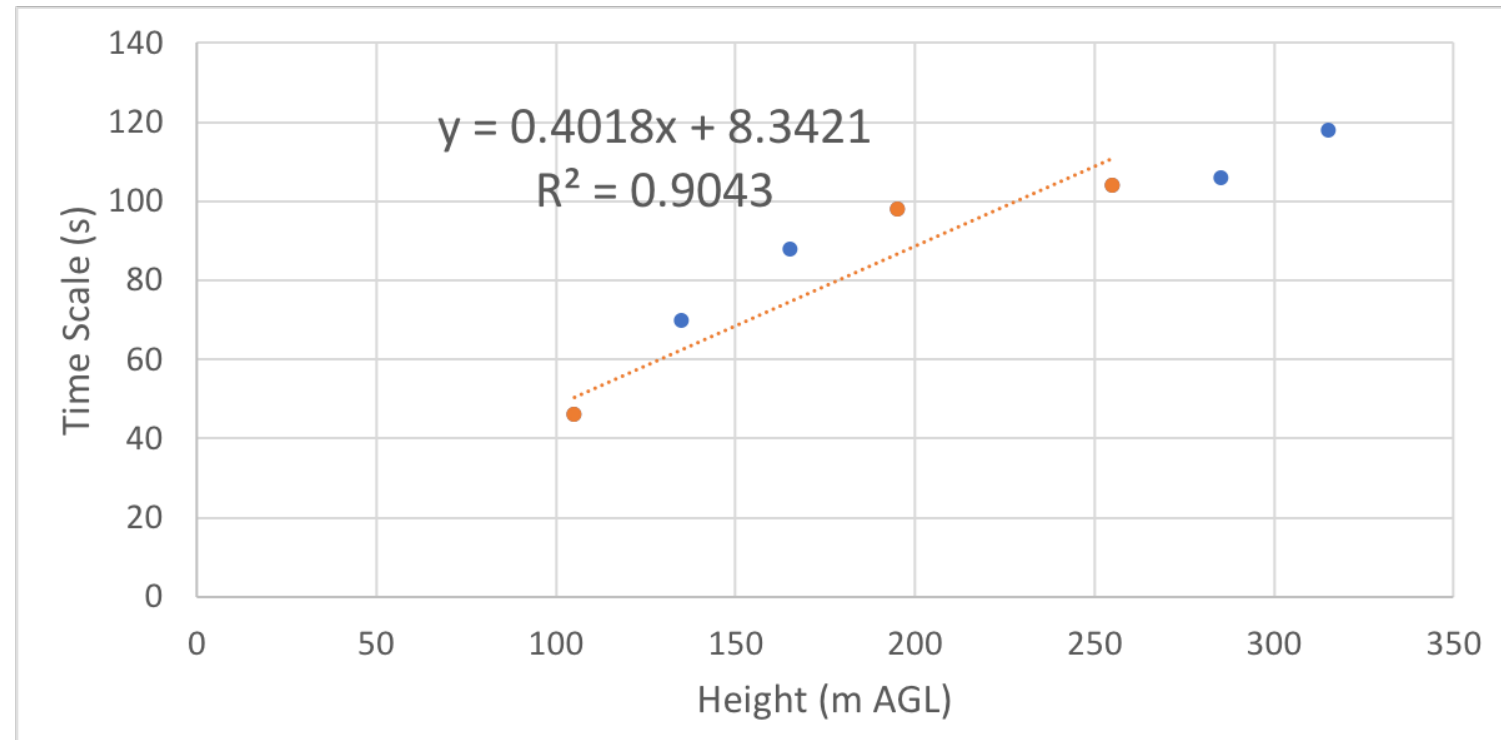


Figure S4: variability of the appropriate sample size with height for the Halo Streamline lidar, during unstable conditions. The best-fit is performed by using the orange dots only, as these correspond to heights with a match with the levels of the sonic anemometers on the BAO tower.

\section{Climatology of turbulence dissipation rate from the WINDCUBE v1s}

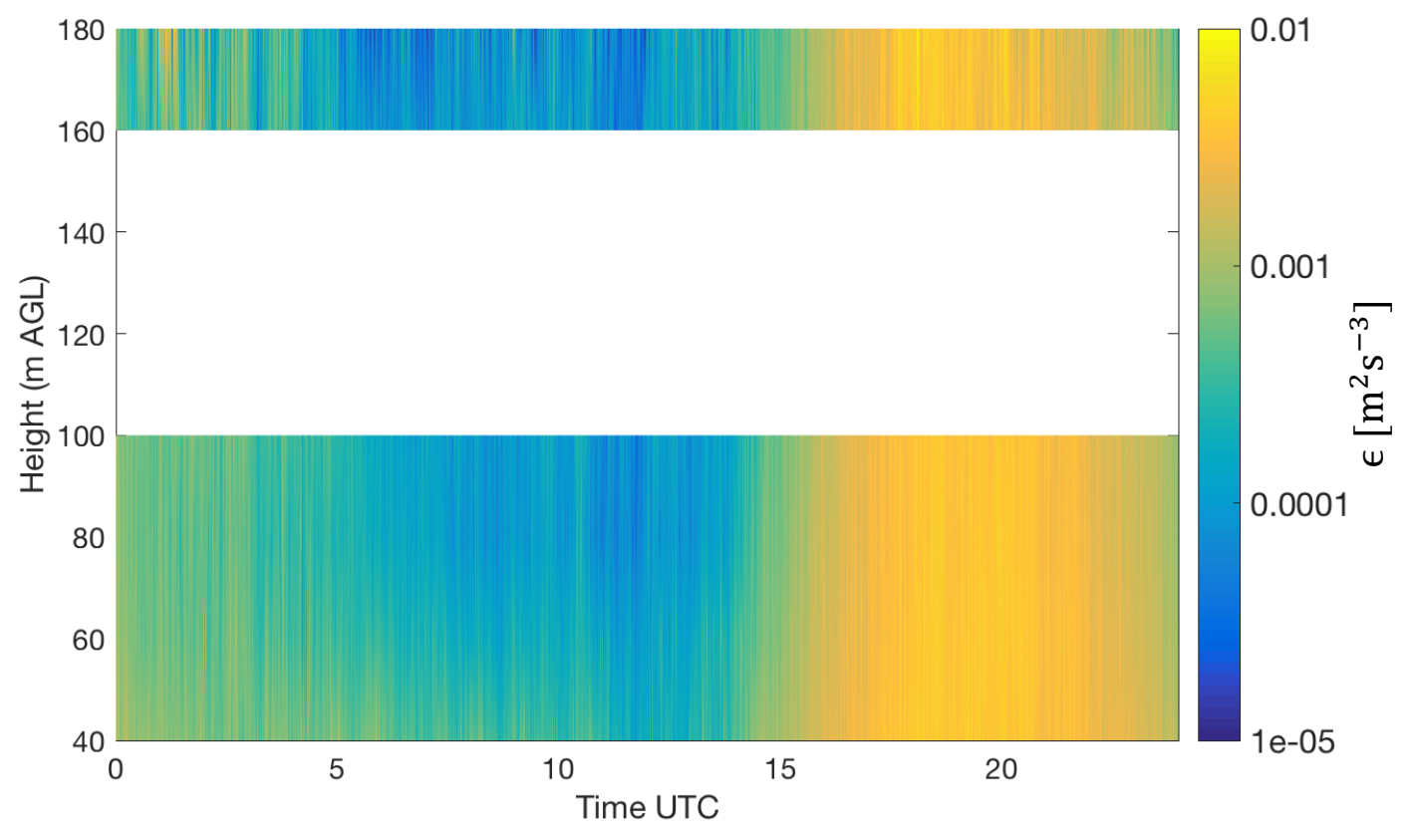

Figure S5: Daily climatology of turbulence dissipation rate derived from the WINDCUBE v1-61 lidar. Data at 120m and 140m AGL are not shown due to hard strikes with the guy wire of the BAO tower. 


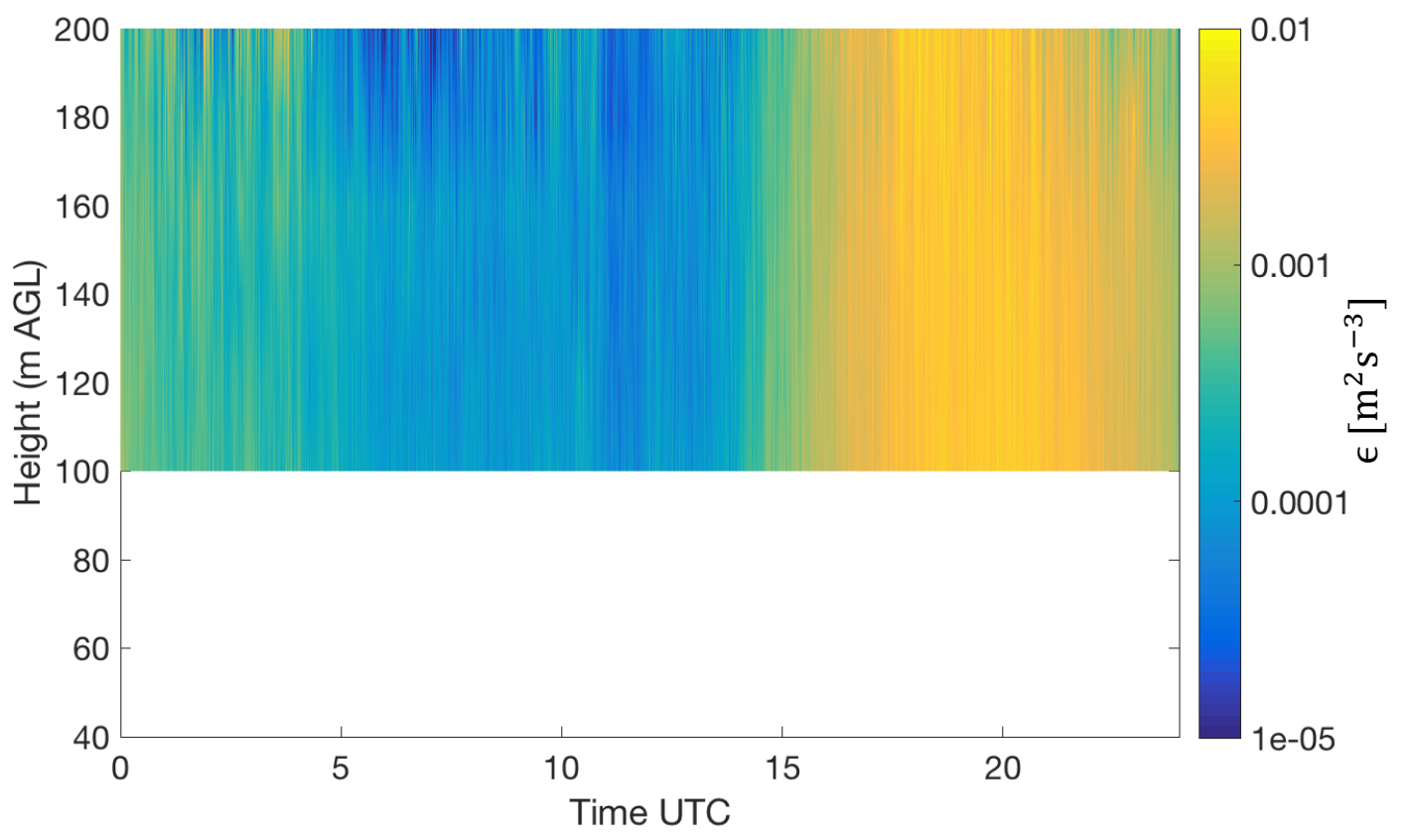

Figure S6: Daily climatology of turbulence dissipation rate derived from the WINDCUBE v1-68 lidar. Data at 40m, 60m and 80m AGL are not shown due to hard strikes with the guy wire of the BAO tower. 


\section{Turbulence dissipation rate as a function of the absolute value of the Obukhov Length from the WINDCUBE v1s}

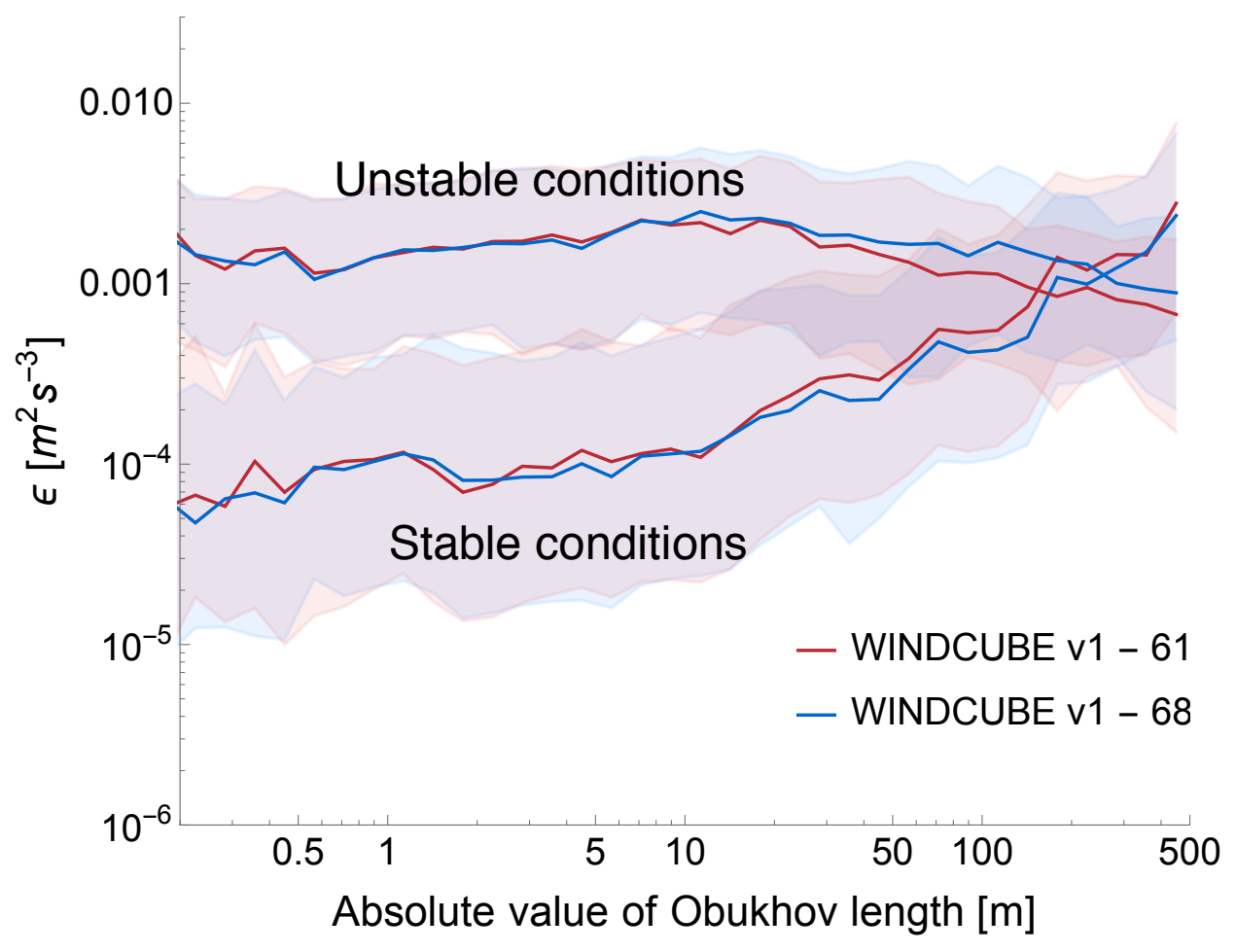

Figure S7: Turbulence dissipation rate (measurements at $100 \mathrm{~m} \mathrm{AGL)}$ as a function of the absolute value of the Obukhov Length, for the WINDCUBE v1s. The continuous lines in the plot represent the median value for the different instruments, while the shaded area creates a band corresponding to the 1st and $3 r d$ quartiles of the distributions. 


\section{Turbulence dissipation rate as a function of wind speed from the WINDCUBE v1s}

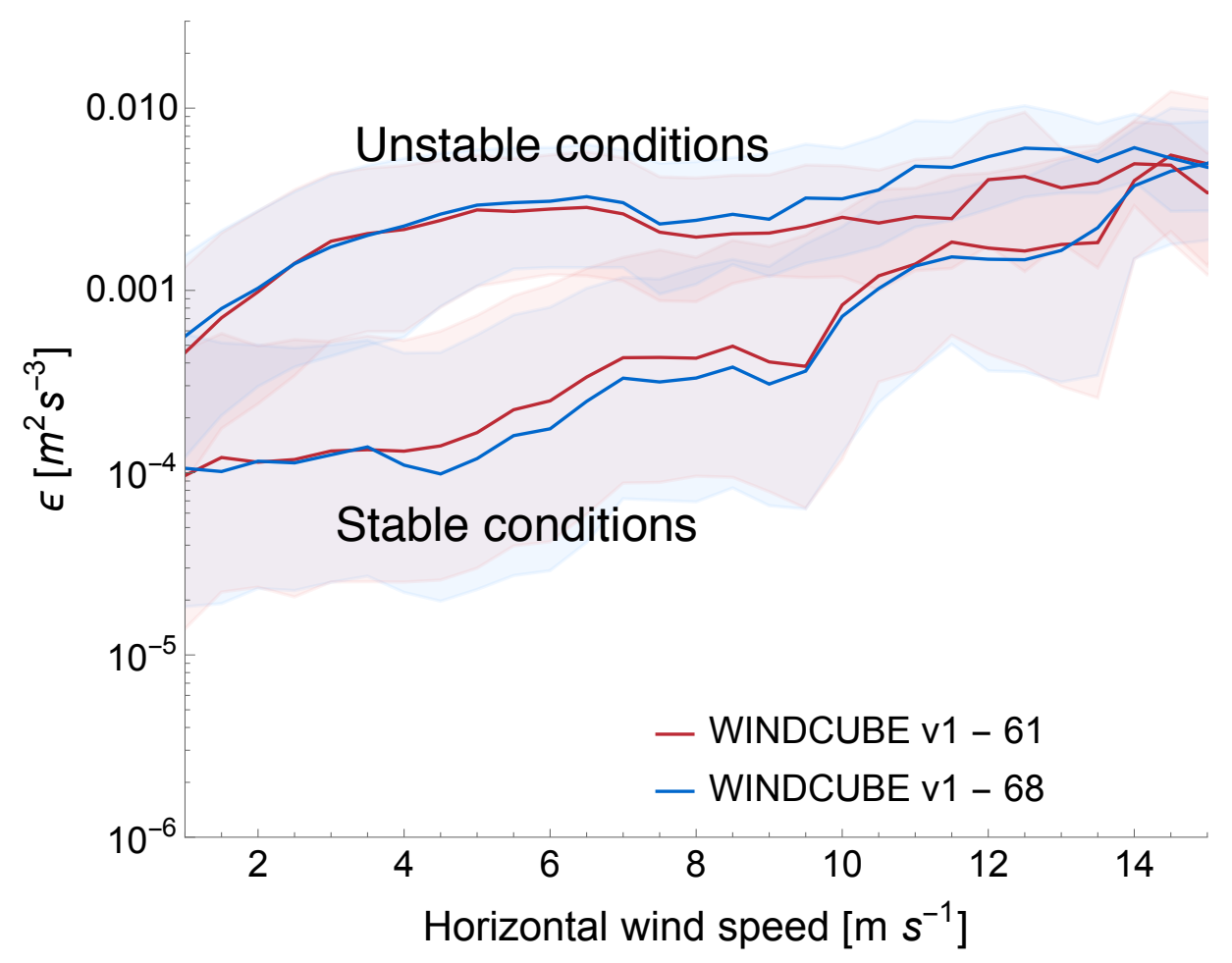

Figure S8: Turbulence dissipation rate as a function of the 2-min average wind speed, as measured at $100 \mathrm{~m} \mathrm{AGL}$, for the WINDCUBE v1s. The continuous lines in the plot represent the median value for the different instruments, while the shaded area creates a band corresponding to the 1st and 3rd quartiles of the distributions. 